\title{
La Variation du /R/ dans L'espagnol de Santiago
}

Orlando Alba

Brigham Young University - Provo, orlando.primero.oa@gmail.com

Follow this and additional works at: https://scholarsarchive.byu.edu/facpub

Part of the Spanish and Portuguese Language and Literature Commons

\section{Original Publication Citation}

1986. "La variation du /r/ dans l'espagnol de Santiago." En Diversity and Diachrony. Editado por David Sankoff. Amsterdam/Philadelphia: John Benjamins Publishing Company. (Current Issues in Linguistic Theory. 53) pp. 211-222.

\section{BYU ScholarsArchive Citation}

Alba, Orlando, "La Variation du /R/ dans L'espagnol de Santiago" (1986). Faculty Publications. 1204. https://scholarsarchive.byu.edu/facpub/1204 accepted for inclusion in Faculty Publications by an authorized administrator of BYU ScholarsArchive. For more information, please contact ellen_amatangelo@byu.edu. 

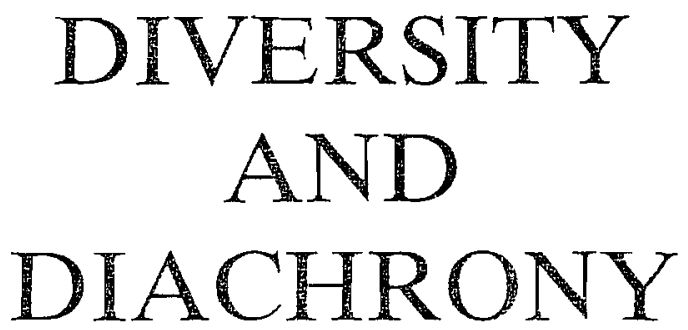

Edited by

\section{DAVID SANKOFF \\ Centre de recherches mathématiques}

Université de Montréal 


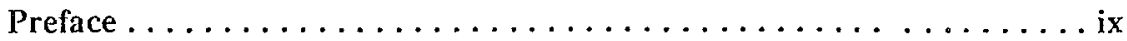

Contributors $\ldots \ldots \ldots \ldots \ldots \ldots \ldots \ldots \ldots \ldots \ldots \ldots \ldots \ldots \ldots \ldots \ldots \ldots$

\section{VARIETIES OF ENGLISH AND THEIR HISTORY}

De Facto Segregation of Black and White Vernaculars

William Labov and Wendell A. Harris ................ 1

The Use of the Verbal -s Inflection in BEV

John Myhill and Wendell A. Harris.............. 25

Linguistic Correlates of Inter-Ethnic Contact

Sharon Ash and John Myhill................ 33

Testing Listeners' Reactions to Phonological Markers of Ethnic Identity:

A New Method for Sociolinguistic Research

David Graff, William Labov and Wendell A. Harris ......... 45

Of-Reduction in Black English: A Quantitative Study

Francisca Sanchez ...................... 59

Contrastive Use of Verbal - $x$ in Slave Narratives

Walter Pitts ..........................73

More Evidence for Major Vowel Change in the South

Crawford Feagin....................... 83

Variation and the Study of English Historical Syntax

Matti Rissanen .........................997

The Development of Preverbal Only in Early Modern English

Terttu Nevalainen.........................111

On the Use of the Modal Auxiliaries Can and May in American English Merja Kytö........................ 123

Is There an Adverbial in this Text? (And If So, What Is It Doing There?)

Gunnel Tottie.......................... 139

Syntactic Development after Childhood: Beyond the Vernacular

Benji Wald........................... 153 


\section{CHANGE AND VARIATION \\ IN ROMANCE}

Going Through (L) in Canadian French

Shana Poplack and Douglas Walker........... 173

/S/ Deletion and Pronoun Usage in Puerto Rican Spanish

Judith G. Hochberg . . . . . . . . . . . . . . . 199

La variation $\mathrm{du} / \mathrm{r} / \mathrm{dan}$ l'espagnol de Santiago

Orlando Alba ..................... 211

Changements en chaine dans le français montréalais

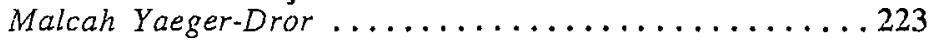

Intonational Variability in Language Contact: $\mathrm{F}_{0}$ Declination

in Ontarian French

Wladyslaw Cichocki and Daniel Lepetit............. 239

Functional and Structural Properties in a Variable Syntax

Fernando Tarallo .................... 249

Variation in Case Marking with Infinitival and Clausal Complements

in Old French

Elizabeth Pearce .................. 261

The Social Profile of a Syntactico-Semantic Variable: Three Verb Forms

in Old Castile

Carmen Silva-Corvalán ................... 279

Metrical Structure and Vowel Deletion in Montreal French

Henrietta J. Cedergren ................... 293

Grammaticalisation des pronoms de la troisième personne en français parlé à Montréal

Pierrette Thibault ................. 301

Variation linguistique: le cas des pronoms personnels du français

Denise Deshaies ...................... 311

Les expressions de la restríction en français de Montréal

France Massicotte ...................... 325

Formes connectives et cohésion textuelle dans le discours conversationne]

d'enfants de différentes classes sociales dans le capitale mexicaine

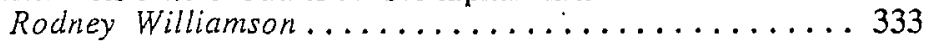

Is Child Language a Possible Source of Linguistic Variation?

Raymond Mougeon, Edouard Beniak and Daniel Valois . . . . 347 


\section{FUNCTIONS AND DISCOURSE}

Linguistic Analysis of the Three Kinds Ralph W. Fasold....................... 361

Turn-Initial Variation: Structure and Function in Conversation Deborah Schiffrin............................. 367

Quantificateur et marqueur de discours Monique Lemieux, Carmen Fontaine and David Sankoff ...... 381

Toward a Unified Model of Sociolinguistic Prestige Edward Finegan and Douglas Biber .............. 391

Cajun/English Code-Switching: A Test of Formal Models Becky Brown ... . . . . . . . . . . . . . . . . . . 399

Factors Affecting the Form of Question Signals in American Sign Language Charlotte Baker-Shenk..................... 407

Constituent-Gap Dependencies in Norwegian: An Acceptability Study Chet A. Creider........................ 415

Author Index ........................... 425 


\title{
LA VARIATION DU /R/ DANS L'ESPAGNOL DE SANTIAGO
}

\author{
Orlando Alba \\ Universidad Católica Madre y Maestra
}

\section{DNTRODUCTION}

1.1 Le segment phonologique /r/ en position implosive montre une grande variété de manifestations phonétiques dans les différents dialectes hispaniques (v. Navarro Tomás 1972; Alarcos Llorach 1968; Zamora Vicente 1970). Parmi les manifestations les plus connues, on peut citer la variante alvéolaire vibrante simple carne 'viande', la fricative carne, l'aspirée cahne, la latéralisée calne, l'assimilée canne et l'élidée cane.

On considère généralement la vibrante comme la réalisation standard, mais la norma culta 'la norme cultivée' admet aussi la prononciation fricative. Toutes les autres prononciations, des points de vue académique et normatif, sont jugées vulgaires, populaires ou régionales (Navarro Tomás 1972:119-121).

1.2 Dans la République Dominicaine le comportement du $/ \mathrm{s} /$ a été étudié par plusieurs auteurs. Henriquez Ureña (1975:147-149) et Jiménez Sabater (1975:88-105) ont énuméré et décrit les différentes transformations subies par le $/ \tau \mid$ dans le parler rural et populaire: l'intermédiaire entre $l$ et $r$, l'aspiration pharyngale, l'assimilation à la consonne suivante, la latéralisation, la vocalisation en [i] et l'élision. Ces deux auteurs, mais tout spécialement Jiménez Sabater, ont présenté un grand nombre de renseignements non quantifiés à partir desquels nous tirons les conclusions suivantes:

a) la variante vocalisée (pueita 'porte') prédomine dans le Cibao, région qui s'étend au nord du pays et à laquelle appartient l'échantillon choisi pour cette étude.

b) à l'est du pays prévaut l'assimilation (puetta).

c) la latéralisation (puelta) est utilisée majoritairement à Santo Domingo, la capitale du pays.

Cependant, plus récemment Rojas (1982:273-285) a étudié quantitativement le phénomène de la vocalisation dans une communauté rurale du Cibao. Il a trouvé un pourcentage global de $30 \%$ de variantes vocalisées, chiffre très inférieur à celui que l'on devait attendre d'après les affirmations et les renseignements non quantifiés d'Henriquez Ureña et de Jiménez Sabater. D'après Rojas (1982:277), dans le dialecte du Cibao la norme (50\% des cas) comporte les mêmes variantes du $/ \mathrm{r} /$ (vibrante ou fricative) que l'espagnol général.

Les trois travaux dont nous avons parlé ci-dessus ont en commun, d'une part, qu'ils décrivent le parler des communautés rurales et, d'autre part, qu'ils n'établissent pas de comparaisons d'ordre sociolinguistique. Seulement deux travaux publiés jusqu'à date traitent de la prononciation du $/ \mathrm{s} / \mathrm{chez}$ des locuteurs urbains appartenant à différentes couches socioculturelles. Jorge Morel (1974), de 
même que Nuñez Cedeño (1980), ont travaillé avec des informateurs de Santo Domingo. Nuñez Cedeño présente la prononciation latérale $d u / r /$ comme une caractéristique des groupes socio-économiques inférieurs. Le groupe de la classe élevée, par contre, utilise les prononciations vibrante et fricative.

1.3 La présente étude analyse des données enregistrées auprès de 12 personnes, choisies d'un total de 35 qui font partie de l'échantillon d'une recherche plus poussée. Toutes habitent à Santiago, la ville la plus importante du Cibao, et la deuxième ville du pays. Huit informateurs sont ouvriers et appartiennent au secteur socio-économique inférieur. Les quatre autres informateurs sont des professionnels ayant un niveau socio-économique élevé. Parmi les huit ouvriers, il y en a quatre qui ont plus de 50 ans; les autres ont moins de 30 ans. Nous avons analysé environ 10 à 15 minutes de conversations spontanées enregistrées pour chacun des informateurs.

Admettant qu'avec un échantillon si réduit on ne peut qu'entamer une étude quantitative de la variation du $/ r /$ à Santiago, nous pouvons espèrer tout au moins en tirer une vision générale et objective, qui servira de point de départ à d'autres recherches plus complètes.

1.4 Parmi les objectifs que nous proposons, nous pouvons citer les suivants:

a) découvrir jusqu'à quel point la variation du $/ \mathbf{r} /$ est un facteur discriminatoire de deux groupes socio-économiques extrêmes.

c) déterminer, à partir de sa fréquence d'apparition, la vitalité réelle de la variante vocalisée dans une région où d'après ce que l'on affirme continuellement, "les gens parlent avec le $\mathrm{i}$ ".

c) analyser l'effet exercé sur la variation $d u / r /$ par deux facteurs linguistiques: la nature du segment phonologique suivant et l'accent de la syllabe à laquelle correspond le $/ r /$.

\section{LES VARIANTES}

La détermination des variantes du/r/ examinées dans ce travail a été réalisée en tenant compte des observations d'études antérieures et, plus particulièrement, en faisant une analyse préliminaire de deux des douze conversations utilisées.

Voici les variantes sélectionnées:

[r] vibrante

[r] fricative

[ri mixte ou intermédiaire entre le $r$ et le son vocalisé

[i] vocalisée

[ø] élidée

[1] latéralisée

[h] aspirée

Il est important de préciser que l'établissement de ces variantes, tout spécialement la variante fricative et la variante mixte ou intermédiaire, comporte certaines simplifications ou standardisations. Nous avons classé dans le groupe des fricatives des réalisations phonétiques qui, dans une analyse plus détaillée, auraient été distinguées d'après la tension musculaire ou la proximité de la langue aux alvéoles. Quoiqu'il en soit, elles se distinguent clairement de la réalisation vibrante, car dans la prononciation de celle-ci se produit un contact apico-alvéolaire, absent lors de 
l'articulation des fricatives.

La variante intermédiaire constitue, elle aussi, un groupe dans lequel nous avons classé des réalisations différentes. Il s'agit en fait d'articulations peu définies, pour lesquelles il est par conséquent souvent difficile de déterminer si c'est l'élément consonantique ou vocalique qui prédomine. Ce qui est clair c'est la distinction entre la variante intermédiare et la fricative, et entre cette variante et le son 'i', tel qu'entendu dans aceite 'l'huile'. Elle présente souvent certaines ressemblances avec le son appelé 'schwa'.

Nous avons transcrit l'élision chaque fois qu'à l'examen auditif (non instrumental) il y avait absence de phonation. En ce qui concerne la variante vocalisée, il est important de noter ici qu'il existe des cas où il est impossible de la confondre avec la semi-voyelle d'un mot tel que soy 'je suis'. En effet, la variante vocalisée est légèrement moins fermée. Toutefois, la perception rapide et naturelle l'identifie ou l'associe à la semi-voyelle [i] plutôt qu'à n'importe quel autre segment espagnol.

\section{RESULTATS GLOBAUX}

\subsection{FACTEUR SOCIO-ECONOMIQUE}

La distribution générale des variantes du $/ r /$ en finale de syllabe et de mot dans les deux groupes socio-économiques étudiés est présentée dans le Tableau 1.

Tableau 1. Distribution, en pourcentages, des variantes de /r/d'après le facteur socio-économique

\begin{tabular}{lrrrr}
\hline & \multicolumn{2}{c}{ COUCHE INFERIEURE } & \multicolumn{2}{c}{ COUCHE ELEVEE } \\
& $\mathbf{N}$ & $\%$ & N & $\%$ \\
\hline $\mathbf{r}$ & 109 & 14 & 167 & 35 \\
$\mathbf{i}$ & 216 & 27 & 235 & 50 \\
$\mathbf{i} \mathbf{i}$ & 80 & 10 & - & - \\
$\mathbf{i}$ & 160 & 20 & - & - \\
6 & 204 & 26 & 62 & 13 \\
$\mathbf{l}$ & 17 & 2 & 9 & 2 \\
$\mathbf{h}$ & 4 & 0.5 & - & - \\
\hline
\end{tabular}

790

473

Le tableau précédent démontre que les deux groupes se distinguent par rapport à la prononciation $\mathrm{du} / \mathrm{t} /$. Tandis que le groupe appartenant à la couche sociale élevée emploie les variantes caractéristiques de l'espagnol général (vibrantes et fricatives) dans $85 \%$ des cas, le groupe appartenant à la couche sociale inférieure le fait à peine dans $41 \%$ des cas. Une proportion similaire mais inverse est observée par rapport à l'élision qui se manifeste deux fois plus dans la couche sociale inférieure que dans la conche sociale élevée.

Toutefois le phénomène le plus intéressant qui ressort du tableau n'est pas la différence quantitative que l'on vient de souligner, mais la distinction qualitative créé par les variantes mixte et vocalisée. Ni l'une ni l'autre n'apparaît dans le dialecte des couches sociales élevées, ce qui augmente considérablement leur valcur 
diagnostique du sociolecte inférieur. Bien qu'aucune de ces deux variantes ne présente une fréquence élevée, leur caractère exclusif aux couches inférieures leur confère une haute signification sociolinguistique étant donné que les différences qualitatives sont plus faciles à percevoir que les différences quantitatives, et sont ainsi plus discriminatoires.

Pour ce qui est de l'élision, il faut souligner que les pourcentages globaux sont relativement importants car le calcul inclut les nombreuses apparitions du complémentiseur porque 'parce que' où, tel que nous le verrons plus loin, le son $/ \mathrm{r} /$ a une forte tendance à l'élision, de même que dans d'autres dialectes espagnols des Caraibes. Si l'on excluait du calcul général les 162 occurrences de cette conjonction, le pourcentage de l'élision serait alors de $15 \%$ dans la couche inférieure et de $6 \%$ dans la couche sociale élevée.

Finalement il est clair que les dernières variantes citées ([h] et [1]) n'ont pas de valeur significative. La variante aspirée a été enregistrée quatre fois dans la couche sociale inférieure, toujours en syllabe tonique intérieure suivie de n: cahne 'viande', à deux reprises, gobiehno 'gouvemement' et piehna 'jambe' une seule fois. La variante latéralisée n'a pas non plus une grande importance: sa fréquence est basse dans les deux sociolectes. D'autre part, dans la plupart des cas elle est le résultat d'une assimilation à la consonne suivante: pol la mañana 'pendant la matinée', arreglallos 'Ies réparer', etc. Nous avons trouvé que 13 des 17 cas du groupe inférieur et 7 des 9 cas du groupe élevé apparaissent devant $/ 1 /$.

L'absence presque totale de la variante latéralisée oppose le dialecte de Santiago à celui de Santo Domingo (Jiménez Sabater 1975; Nuñez Cedeño 1979) et à celui de San Juan, Puerto Rico (López Morales 1982) où la latéralisation est un phénomène important. Cependant, cette absence coïncidence avec l'espagnol de Panamà, d'après les données de Cedergren (1973), et avec le sociolecte cubain 'cultivé', d'après Terrell (1978).

\subsection{FACTEUR GENERATIONNEL}

D'autre part, l'analyse du /r/ nous a permis de constater que le facteur générationnel marque aussi une différence appréciable à l'intérieur du groupe socio-économique bas.

Tableau 2. Distribution en pourcentages des variantes de $/ \mathbf{r} /$ à i'intérieur du groupe social inférieur, selon l'âge

\begin{tabular}{lrc}
\hline & $\begin{array}{c}\text { AGÉS } \\
\%\end{array}$ & $\begin{array}{c}\text { JEUNES } \\
\%\end{array}$ \\
\hline $\mathbf{r}$ & & \\
\hline $\mathbf{7}$ & 6 & 22 \\
$\mathbf{i} \mathbf{i}$ & 16 & 38 \\
$\mathbf{i}$ & 5 & 15 \\
$\mathbf{6}$ & 38 & 3 \\
$\mathbf{l}$ & 31 & 21 \\
$\mathrm{~h}$ & 3 & 1 \\
\hline
\end{tabular}

$\mathrm{N}$ : 
D'après les données du Tableau 2 , la prononciation standard (vibrante et fricative) prédomine chez les jeunes ( $60 \%$ des cas), tandis que chez les vieux elle ne représente que $22 \%$. Les personnes âgées manifestent au contraire plus fréquemment la variante vocalisée $(38 \%)$ face à un mince $3 \%$ chez les jeunes qui utilisent davantage la variante intermédiaire.

Bien qu'à ce sujet la différence ne soit pas qualitative, elle est suffisamment grande pour montrer quelques aspects intéressants de la vocalisation. Les locuteurs de la nouvelle génération, qui expérimentent une mobilité sociale majeure et un contact plus continuel et direct avec d'autres niveaux socio-économiques, surtout à l'école, ont souvent tendance à substituer la vocalisation par la variante intermédiaire. Par contre les âgés, plus conservateurs et moins exposés à l'influence des couches élevées ou de l'école, conservent la variante dans une proportion relativement significative.

Selon nous, la constatation précédente souligne d'une façon très claire la grande censure sociale ou le stigma qui porte sur la vocalisation. En ce sens, nous pouvons penser qu'il s'agit d'un phénomène linguistique qui, peu à peu, a commencé un processus de régression. Une situation similaire a été découverte par López Morales (1982) pour l'espagnol de San Juan, par rapport à la latéralisation. La jeune génération défavorise l'application de la règle, ce qui permet au chercheur de soupçonner une réduction prochaine du phénomène.

\section{4. /r/ A L'TNTERIEUR DU MOT}

Dans le calcul du $/ \mathrm{r} /$ implosif à l'intérieur du mot (Tableau 3 ), on a décidé de mettre à part la conjonction porque 'parce que', tel que l'ont fait d'autres chercheurs (D'Introno 1979; Rojas 1982), puisqu'elle présente un comportement spécifique fortement favorable à l'élision qui est dû, sans aucun doute, à son emploi très fréquent.

Tableau 3. Distribution des variantes du $/ \mathrm{r} /$ dans "porque"

\begin{tabular}{lcc}
\hline & $\begin{array}{c}\text { COUCHE INFERIEURE } \\
\%\end{array}$ & COUCHE ELEVEE \\
$\%$
\end{tabular}

La très grande fréquence d'élision $d u / \tau /$ dans cette conjonction nous permet de supposer que, dans ces dialectes, il se produit en fait une réorganisation phonologique du mot sous la forme poke.

Ainsi, notre attention est particulièrement attirée par le fait que les deux sociolectes coïncident presque exactement dans l'application de la règle de l'élision pour ce cas concret qui, tel que D'Introno (1979:76) et Rojas (1982:279) l'ont signalé, a un caractère morphologique et non phonologique. Autrement dit, le /r/ est élidé en prenant compte du mot lui-même et de sa catégorie et non pas de son 
contexte phonétique. En effet, cette interprétation est appuyée par le fait que la séquence [rk], dans d'autres mots, ne présente pas un taux d'élision si élevé.

L'élision de $/ \mathrm{I} /$ dans porque dans l'espagnol de Caracas (D'Introno 1979:63), diffère de l'espagnol de Santiago en distinguant les deux groupes socio-économiques. Le groupe appartenant à la classe inférieure présente $70 \%$ d'élision et la classe élevée, $38 \%$.

Le Tableau 4 montre la situation globale de $/ \mathbf{z} /$ à l'intérieur du mot, en excluant le mot porque.

Tableau 4. Variantes de /r/à l'intérieur du mot

\begin{tabular}{lcc}
\hline & $\begin{array}{c}\text { COUCHE INFERIEURE } \\
\%\end{array}$ & $\begin{array}{c}\text { COUCHE ELEVEE } \\
\%\end{array}$ \\
\hline$r$ & 9 & 32 \\
$\mathbf{1}$ & 39 & 64 \\
$\mathbf{i}$ & 11 & - \\
6 & 25 & 1 \\
$\mathrm{I}$ & 10 & 3 \\
$\mathrm{~h}$ & 4 & - \\
\hline $\mathrm{N}$ & 2 & 179
\end{tabular}

Si on considère, parmi cet ensemble, les cas d'infinitif suivi d'un clitique, on obtient le tableau suivant:

Tableau 5. Variantes de /r/à l'infinitif suivi d'un clitique

\begin{tabular}{lcc}
\hline & $\begin{array}{c}\text { COUCHE INFERIEURE } \\
\%\end{array}$ & $\begin{array}{c}\text { COUCHE ELEVEE } \\
\%\end{array}$ \\
\hline $\mathrm{r}$ & 16 & 36 \\
$\mathrm{i}$ & 27 & 48 \\
$\mathrm{i} \mathrm{i}$ & 11 & - \\
$\emptyset$ & 21 & - \\
$\mathrm{i}$ & 13 & - \\
\hline $\mathrm{N}:$ & 12 & 31
\end{tabular}

Il est nécessaire de préciser que toutes les réalisations latéralisées dans les deux groupes ont lieu devant [1]. En fait, il ne s'agit pas d'une latéralisation proprement dite, mais plutôt d'une assimilation à la consonne suivante. Par conséquent, nous pouvons dire que l'espagnol de Santiago n'a pas de règle indépendante de latéralisation de $/ \mathrm{r} /$.

Les autres cas de /r/ à l'intérieur du mot (sauf porque et l'infinitif suivi d'un clitique) ont été analysés en tenant compte de l'effet possible de deux facteurs linguistiques: le segment phonologique suivant et l'accent de la syllabe où le $/ \mathrm{r} /$ 
apparaît.

Le Tableau 6 montre les résultats obtenus selon le segment suivant. On n'inclut pas le contexte pré-latéralisé, car il ne s'est présenté qu'en deux occasions.

Tableau 6. Variantes de $/ \mathbf{r} /$ à l'intérieur du mot d'après le segment phonologique suivant

\begin{tabular}{|c|c|c|c|c|c|c|}
\hline & \multirow{2}{*}{$\begin{array}{l}\text { COUCHE } \\
\text { Occlusive }\end{array}$} & \multicolumn{2}{|c|}{ SOCIALE INFERIEURE } & \multicolumn{3}{|c|}{ COUCHE SOCLALE ELEVEE } \\
\hline & & Fricative & Nasale & Occlusive & Fricative & Nasale \\
\hline $\mathbf{r}$ & $4 \%$ & $9 \%$ & $5 \%$ & $39 \%$ & $28 \%$ & $32 \%$ \\
\hline $\mathbf{r}$ & 44 & 49 & 41 & 58 & 71 & 63 \\
\hline $\mathbf{r} \mathbf{i}$ & 17 & 9 & 8 & - & - & - \\
\hline i & 30 & 24 & 30 & - & - & - \\
\hline$\emptyset$ & 5 & 9 & 5 & 3 & 1 & - \\
\hline h & - & - & 11 & - & - & - \\
\hline 1 & - & - & - & - & $=$ & 5 \\
\hline$N$ & 54 & 66 & 37 & 33 & 96 & $\overline{19}$ \\
\hline
\end{tabular}

Bien que la variante fricative soit privilegiée dans les trois contextes analysés, sa présence est légèrement plus favorisée devant une consonne fricative. Les rares occurrences de la variante aspirée ne sont possibles que devant une nasale, tandis que la variante mixte ou intermédiaire est plus fréquente devant une occlusive. Une fricative qui suit le $/ r$ / défavorise légèrement sa vocalisation. Enfin, l'élision est rare en position intérieure, quel que soit le contexte.

Dans le dialecte portoricain de Philadelphie étudié par Poplack (1979:155-158), l'aspiration du /r/ est favorisée par une nasale qui le suit, tandis que les occlusives ont tendance à provoquer la latéralisation. A San Juan, Puerto Rico (López Morales 1982), la latéralisation est aussi provoquée par un contexte pré-occlusif ou pré-fricatif, alors qu'une nasale qui suit favorise la variante fricative.

Finalement, les données ont été classées d'après la présence ou l'absence d'accent dans la syllabe comportant le $/ \mathrm{r} /$.

Tableau 7. Variantes dud/ $/$ /à l'intérieur du mot d'après.l'accent

\begin{tabular}{|c|c|c|c|c|}
\hline & \multicolumn{2}{|c|}{ COUCHE SOCIALE INFERIEURE } & \multicolumn{2}{|c|}{ COUCHE SOCLAIE ELEVEE } \\
\hline & Syllabe & Syllabe & Syllabe & Syllabe \\
\hline & Accentuée & Inaccentuée & Accentuée & Inaccentuée \\
\hline $\mathbf{r}$ & $8 \%$ & $5 \%$ & $42 \%$ & $24 \%$ \\
\hline$z^{2}$ & 41 & 49 & 56 & 74 \\
\hline $\mathbf{i} \mathbf{i}$ & 16 & 7 & - & - \\
\hline i & 28 & 27 & - & - \\
\hline $\mathfrak{\wp}$ & 2 & 12 & - & 2 \\
\hline $\mathbf{h}$ & 5 & - & - & - \\
\hline 1 & - & - & 2 & - \\
\hline $\mathrm{N}:$ & 76 & 82 & 59 & 89 \\
\hline
\end{tabular}


Dans le sociolecte inférieur il faut souligner que l'absence d'accent dans la syllabe favorise quelque peu l'élision ( $12 \%$ face à $2 \%$ ) et la variante fricative ( $49 \%$ en syllabe atone et $41 \%$ en syllabe tonique). En revanche, l'accent favorise la variante mixte et l'aspirée. Celle-ci n'apparaît qu'en syllabe tonique devant [n].

Dans le sociolecte élevé, l'accent contribue à la réalisation vibrante ( $42 \%$ en syllabe tonique et $24 \%$ en syllabe atone), tandis que la fricative prédomine en syllabe inaccentuée ( $74 \%$ en syllabe atone et $56 \%$ en syllabe tonique). Ces résultats sont prévisibles: face à l'absence du renforcement par accent, l'articulation de la vibrante tend à se relâcher en donnant lieu à une fricative.

Dans le dialecte portoricain de Philadelphie, Poplack (1979:160-161) découvre que quand la syllabe suivant le son / $/$ / est accentué, c'est-à-dire quand /r/ se trouve en syllabe atone, son affaiblissement est significativement favorisé sous la forme d'une assimilation à la consonne suivante ou de l'élision.

\section{5. /r/FTNAL DE MOT}

5.1 Le Tableau 8 montre les résultats de la variation de $/ r /$ à la fin du mot.

Tableau 8. Distribution des variantes du/r/à la fin du mot

\begin{tabular}{lcc} 
& $\begin{array}{c}\text { COUCHE INFERIEURE } \\
\%\end{array}$ & $\begin{array}{c}\text { COUCHE ELEVEE } \\
\%\end{array}$ \\
\hline $\mathbf{r}$ & 19 & 43 \\
$\mathbf{i}$ & 27 & 47 \\
$\mathrm{r} \mathbf{i}$ & 12 & - \\
$\mathbf{i}$ & 23 & - \\
$\sigma$ & 18 & 1 \\
\hline $\mathbf{l}$ & 1 & 256
\end{tabular}

Si nous comparons les résultats précédents avec ceux du Tableau 4 concernant $\mathrm{le} / \mathrm{r} / \mathrm{a}$ l'intérieur du mot, on constate que les différentes positions (intérieure: arma 'arme' ou finale: amar 'aimer') occupées par le segment influent visiblement sur sa réalisation. Dans les deux groupes, la position en finale de mot favorise la conservation de la vibrante ainsi que l'élision complète. Par contre, la fricative est plus fréquente en position interieure. La position à l'intérieur du mot.produit un effet similaire sur la variation du /s/ dans plusieurs dialectes des Caraỉbes: à l'intérieur du mot l'aspirée [h] est plus fréquente et à la fin, la sibilante [s] et l'élidée [ $\varnothing]$ le sont.

\section{$5.2 / \mathrm{r} /$ A LA FIN DE LINFINITLF ET NON MORPHOLOGIQUE}

Le $/ \mathrm{r} /$ en position finale de mot peut avoir une valeur morphologique en tant que marque d'infinitif: comer 'manger', jugar 'jouer'. Il est dépourvu de cette valeur quand il fait partie d'un morphème unique: amor 'amour' par 'paire'. Nous l'appellerons, dans le premier cas, $/ \mathrm{r} /$ infinitif $\mathrm{et}$, dans le second, $/ \mathrm{r} /$ non morphologique.

Le Tableau 9 nous permet de comparer les résultats des deux catégories dans la couche sociale élevée. 
Tableau 9. Distribution des yariantes de $/ \mathbf{r} /$ dans la couche sociale élevée d'après son statut grammatical

\begin{tabular}{lcc}
\hline & DNFINITIF & NON MORPHOLOGIQUE \\
\hline $\mathrm{r}$ & $43 \%$ & $43 \%$ \\
$\mathrm{I}$ & 44 & 55 \\
$\mathrm{I}$ & 1 & 1 \\
& 12 & 1 \\
\hline $\mathrm{N}:$ & 178 & 78
\end{tabular}

Selon ce qu'on peut observer, le $/ \tau /$ infinitif s'élide plus, ce qui marque une coïncidence entre l'espagnol de Santiago, le panaméen (Cedergren 1973) et l'espagnol de San Juan (López Morales 1982). De plus, la variante fricative est plus fréquente dans $\mathrm{le} / \mathrm{r} /$ non morphologique. Ce comportement semble contredire le principe fonctionnel formulé par Kiparsky (1972:195) puisque le / $/ \mathrm{d}$ de l'infinitif est une marque grammaticale. Cependant, il n'en est rien. Cedergren (1973:111) a démontré que le $/ \mathrm{r} /$ infinitif est une marque sémantique vide qui est prévisible du contexte. Poplack (1979:169) attire l'attention sur le fait que l'accent final de l'infinitif le distingue de toute autre forme verbale avec laquelle on pourrait le confondre, au cas où il perdait le /r/. Ex: amár 'aimer', áma 'il aime'.

La variation du / $/$ / final dans la couche sociale inférieure, en tenant compte du segment phonique suivant et de son statut grammatical, est présentée dans le Tableau 10:

Tableau 10. Distribution des variantes de $/ r /$ dans la couche sociale inférieure selon le seginent suivant et le statut grammatical

Occlusif Fricatif Latéral Nasal Voyelle Pause

\begin{tabular}{lrrrrrr} 
EINAL NON & MORPHQLOGIOUE & & & & \\
\hline $\mathrm{r}$ & - & - & - & $8 \%$ & $78 \%$ & - \\
$\mathrm{i}$ & $44 \%$ & $40 \%$ & $60 \%$ & $50 \%$ & $10 \%$ & $43 \%$ \\
$\mathrm{i} \mathrm{i}$ & $11 \%$ & - & $7 \%$ & $25 \%$ & $2 \%$ & $26 \%$ \\
$\mathrm{i}$ & $39 \%$ & $53 \%$ & $13 \%$ & $17 \%$ & $4 \%$ & $26 \%$ \\
$\phi$ & $6 \%$ & $7 \%$ & - & - & $4 \%$ & $5 \%$ \\
$\mathrm{l}$ & - & - & $20 \%$ & - & $2 \%$ & - \\
\hline TOTAUX: & 18 & 15 & 15 & 12 & 50 & 23
\end{tabular}

EINAL D'INEINITIE

\begin{tabular}{lrrrrrr}
$\mathrm{r}$ & $4 \%$ & $8 \%$ & $13 \%$ & $6 \%$ & $26 \%$ & $15 \%$ \\
$\mathrm{i}$ & $37 \%$ & $33 \%$ & $20 \%$ & $6 \%$ & $20 \%$ & $23 \%$ \\
$\mathrm{i} \mathrm{i}$ & $11 \%$ & $8 \%$ & $20 \%$ & $25 \%$ & $13 \%$ & $10 \%$ \\
$\mathrm{i}$ & $17 \%$ & $24 \%$ & $34 \%$ & $32 \%$ & $13 \%$ & $33 \%$ \\
& $31 \%$ & $27 \%$ & $13 \%$ & $31 \%$ & $28 \%$ & $19 \%$ \\
\hline & & & & & & \\
\hline TOTAUX: & 46 & 37 & 15 & 16 & 79 & 103
\end{tabular}


Ces chiffres nous indiquent que le $/ \mathrm{r} /$ dans les deux catégories de mots se manifeste d'une manière plutôt différente. Premièrement, l'élision est plus fréquente à l'infinitif, de même que dans le sociolecte élevé. Le même effet existe à Panama (Cedergren 1973), à San Juan, Puerto Rico (López Morales 1982) et à Caracas (D'Introno 1979).

D'autre part, la vibrante est favorisée devant une voyelle, surtout quand elle n'est pas morphologique. Le même résultat est enregistré par Cedergren (1973) à Panamà et par Poplack (1979) à Philadelphie.

L'explication de ce phénomène réside dans le fait que plus de la moitié des /I/ non-morphologiques correspondent à la préposition por 'par', mot inaccentué qui adhère étroitement à la voyelle suivante en formant une unité phonétique où le $/ \mathrm{r} /$ devient un élément initial de syllabe: por eso'à cause đe cela ${ }^{\prime}=$ po - re - so, por otro 'par autrui' = po - ro - tro. Il arrive la même chose avec le segment/s/ dans le contexte suivant: los otros 'les autres' = lo - so - tros (Alba 1982).

Parmi les non-morphologiques il est intéressant de découvrir que le processus de vocalisation est favorisé par le contexte pré-fricatif. Par contre, quand le segment suivant est occlusif, latéral, nasal ou une pause, l'apparition de la variante fricative est favorisée. Le $/ r /$ infinitif est vocalisé plus fréquemment devant un son latéral, nasal ou devant une pause que devant un son occlusif, fricatif ou une voyelle.

\section{CONSIDERATIONS PHONOLOGIQUES}

Les processus phonétiques qui mènent de la forme sous-jacente vibrante de $/ \mathrm{s} /$ jusqu'à l'élision totale [ø] sont plus complexes et difficiles à expliquer que le procédé par lequel /s/ se réduit à [ $\emptyset$ ]. Certes, tel que l'explique Poplack (1979:144), il existe non seulement une grande yariété de réalisations, mais aussi des modèles distributionnels qui sont plus compliqués.

Lòpez Morales (1982), Poplack (1979) et Nùnez Cedeño (1979) ont mis en évidence le fait que la latéralisation constitue un processus différent qui ne peut pas être intégré à la chaîne d'affaiblissement de /r/. En effet, il n'y a aucune raison justifiant que [1] constitue un affaiblissement phonétique par rapport à [r]. Par conséquent, il serait nécessaire de formuler deux processus indépendants:

1. latéralisation : $I \rightarrow 1$

2. affaiblissement : $\quad \mathrm{r} \rightarrow \grave{\mathrm{r}} \rightarrow \varnothing$

Quoiqu'il en soit, pour l'espagnol de Santiago le problème ne se pose pas car, tel que nous l'avons indiqué plus haut, la latéralisation est quasi inexistante.

L'aspiration, elle aussi insignifiante dans nos données, est conceptualisée par plusieurs auteurs comme une étape dans le processus d'affaiblissement situé entre la fricative et le $\varnothing$ :

$$
\mathrm{r} \rightarrow \grave{\mathrm{r}} \rightarrow \mathrm{h} \rightarrow \emptyset
$$

Les rares cas enregistrés dans l'espagnol de Santiago rendent inutile cette étape intermédiaire.

Néanmoins pour le dialecte inférieur il faut introduire deux variantes intermédiaires $(r i, i)$; ainsi le processus de réduction du $/ r /$ comprend quatre étapes.

Dans le sociolecte élevé, tel que nous l'avons déjà signalé, ce processus ne comporte pas deux étapes intermédiaires et peut être exprimé de cette façon: 


$$
\mathrm{r} \rightarrow \grave{\mathrm{r}}-\varnothing
$$

Finalement, en ce qui concerne la variante vocalisée, il faudrait bien poser le problème à savoir s'il existe ou pas des preuves qui mettent en évidence qu'elle dérive d'un $/ \mathrm{r} /$ sous-jacent. Sans trop entrer dans les détails, le fait que dans la formation du pluriel le /I/ réapparaisse systématiquement constitue un argument de poids en faveur de cette interprétation.

$$
\begin{aligned}
& \text { mujer (singulier) - mujeres (pluriel) } \\
& \text { 'femme' }
\end{aligned}
$$

\section{CONCLUSIONS}

\subsection{FACTEURS LINGUISTIQUES}

Trois facteurs linguistiques influencent à différents degrés les processus d'affaiblissement et d'élision du /r/ implosif à Santiago: la position dans le mot, le segment phonologique suivant et l'accent.

A la fin du mot les variantes vibrante et élidée sont plus favorisées qu'en position interne. Dans celle-ci, la fricative apparaît plus fréquemment.

Le $/ \mathrm{r} /$ interne est réalisé comme une fricative de préférence dans le contexte où une fricative suit. La vocalisation ne semble être conditionnée spécifiquement par aucun contexte. Le $/ \pi /$ final est notamment retenu en tant que vibrant quand il suit un mot qui commence par une voyelle.

Pour ce qui est de la contribution de l'accent, il est important de souligner que sa présence favorise la prononciation de la vibrante, tandis que son absence entraîne une augmentation de la fricative et de l'élision.

\subsection{FACTEURS SOCIAUX}

Tout an long de l'analyse, on a démontré que le facteur socio-économique exerce une influence dans la variation du $/ \mathrm{r} /$, de telle façon que celui-ci est diagnostique de la condition sociale du locuteur. Les individus du sociolecte élevé se caractérisent par un emploi abondant des réalisations vibrante et fricative, ce qui coïncide avec les groupes du même niveau dans d'autres régions hispanophones. La couche sociale inférieure, quant à elle, tout en réduisant significativement l'utilisation de la vibrante et de la fricative, emploie avec des fréquences plus ou moins élevées des variantes exclusives qui l'identifient clairement: la vocalisée et la mixte.

Contrairement à la croyance généralisée dans le pays et corroborée par les affirmations d'Henriquez Ureña (1975) et de Jiménez Sabater (1975) que la vocalisation du $/ \mathrm{r} /$ implosif est systématique et majoritaire d'une manière accablante dans le Cibao, nos données quantifiées concernant le sociolecte inférieur de Santiago attribuent au phénomène une fréquence de $20 \%$. Ce résultat est consistant avec le $30 \%$ obtenu par Rojas (1982), en considérant qu'il a étudié le parler d'une communauté rurale.

L'impression qu'il s'agit d'un phénomène catégorique et absolu est fondée sans doute sur le fait qu'aucun autre dialecte hispanique présente ce phénomène, du moins avec les mêmes proportions. De plus, parmi la population de Santiago, l'appartenance exclusive de cette variante à un groupe lui prête l'image d'une caractéristique qualitative et non pas purement quantitative. 
Finalement le facteur générationnel apporte également un effect appréciable dans le développement de ces processus. Les jeunes du sociolecte inférieur ne favorisent pas l'emploi de la vocalisation et ils utilisent davantage que les vieux les prononciations de la vibrante, de la fricative et d'une variante intermédiaire entre le son consonantique et le son vocalique. Cette situation chez les locuteurs urbains pourrait présager une diminution prochaine, mais sans doute lente, du phénomène stigmatisé de la vocalisation des liquides.

\section{REEERENCES}

Alarcos Llorach, Emilio. 1968. Fonologia española. Madrid: Gredos.

Alba, Orlando. 1979. Análisis fonológico de las líquidas implosivas en un dialecto rural de la República Dominicana. Boletín de la Academia Puertorriqueña de la Lengua Española. VII 2.1-18.

Alba, Orlando. 1979. Estratificación social del español de Santiago. Variación de la /s/implosiva. Mémoire de maîtrise. Université de Puerto Rico.

Cedergren, Henrietta. 1979. The interplay of social and linguistic factors in Panama. Thèse de doctorat. Université Cornell.

D'Introno, Francesco, N. Rojas et J. Sosa. 1979. Estudio sociolinguístico de las líquidas en posición final de sílaba y final de palabra en el español de Caracas. Boletín de la Academia Puertorriqueña de la Lengua Española. VII 2.59-100.

Henríquez Ureña, Pedro. 1975. El español en Santo Domingo. Santo Domingo: Editora Tailer.

Jiménez Sabater, Maximiliano A. 1975. Más datos sobre el español de la República Dominicana. Santo Domingo: Ed. INTEC.

Jorge Morel, Elercia. 1974. Estudio linguístico de Santo Domingo. Santo Domingo: Editora Taller.

Kiparsky, Paul. 1972. Explanation in phonology. Goals of linguistic theory, dir. par Stanley Peters, 189-227. New Jersey: Prentice Hall.

Labov, William. 1963. The social motivation of a sound change. Word. 19.273-309.

Labov, William. 1966. The social stratification of English in New York City. Washington, D.C. Center for Applied Linguistics.

López Morales, Humberto. 1982. Estratificación social del Español de San Juan. Mimeo.

Navarro Tomás, Tomás. 1972. Mañual de pronunciación española. Madrid: CSIC.

Núñez Cedeño, R. 1980. La fonología moderna y el español de Santo Domingo: Editora Taller.

Poplack, Shana. 1979. Function and process in a variable phonology. Thèse de doctorat. Université de Pennsylvanie.

Rojas, Nelson. 1982. Sobre la semivocalización de las líquidas en el español cibaeño. El español del Caribe, dir. par Orlando Alba. Santiago: Universidad Católica Madre y Maestra.

Terrell, Tracy. 1978. La variación fonética de $/ \mathrm{r} /$ y $/ \mathrm{rr} /$ en el español cubano. Mimeo.

Vallejo-Claros, Bernardo. 1970. La distribución y estratificaciéon de $/ \mathrm{r} / \mathrm{g} / \mathrm{r} / \mathrm{y} / \mathrm{s} /$ en el español cubano. Thèse de doctorat. Université de Texas, Austin.

Zamora Vincente, Alonso. 1970. Dialectología española. Madrid: Gredos. 